\title{
Análisis citogenético en linfocitos de trabajadores expuestos a radiación ionizante en una Unidad de Cardiología Intervencional de Chile: Estudio piloto y revisión de la literatura.
}

\author{
Sergio Ramos-Avasola ${ }^{1, a, e}$, Daniel Rivera ${ }^{1, d}$, Kevin Segura $^{1, d}$, Edgar Thraves $^{1, d}$, Ariel Durán $^{2, b}$, Víctor Soto ${ }^{3, a}$, Jorge Gamarra ${ }^{4, c, f}$, \\ María Isabel Ojeda ${ }^{1, a}$.
}

1. Universidad de Viña del Mar, Viña del Mar, Chile, Facultad de Ciencias de La Salud, Escuela de Tecnología Médica, Agua Santa 7055 , sector Rodelillo, Viña del Mar. Chile.

2. Hospital de Clínicas de la Facultad de Medicina de Montevideo, Universidad de la República (UDELAR), Uruguay.

3. Laboratorio de Citogenética de Hospital Dr. Gustavo Fricke, Viña del Mar, Chile.

4. Comisión Chilena de Energía Nuclear, Santiago, Chile.

a. Tecnólogo Médico, b. Médico, c. Físico, d. Alumno, e. Magíster en Epidemiología, f. Magíster en Biofísica Médica.

Fuente de financiamiento: Fondos de investigación Para alumnos de pre y post grado

Universidad Viña del Mar

Antecedentes: Un número creciente de artículos está llamando la atención en forma consistente sobre la eventual asociación que existe entre los denominados trabajadores ocupacionalmente expuestos a bajos niveles de radiación ionizante (POEs) y una mayor frecuencia de aberraciones cromosómicas, a nivel Sudamericano estos estudios son escasos.

Objetivo: Evaluar la frecuencia de aberraciones cromosómicas en linfocitos de sangre periférica de POEs de un hospital y de sujetos sanos. Adicionalmente, se realizó una revisión exhaustiva de los artículos que a la fecha abordaron este tema.

Material y método: Se condujo un análisis citogenético destinado a cuantificar las aberraciones cromosómicas en sangre periférica de linfocitos de 6 POEs de la unidad de Cardiología Intervencional y, como controles, 6 muestras de sujetos de la población general fueron analizadas.

Resultados: Se observó un importante contraste en el número de aberraciones cromosómicas presentadas en los POEs versus la población general no expuesta a radiaciones ionizantes, siendo esta de una relación de 6:1, respectivamente.

Conclusión: Los resultados preliminares indican una mayor frecuencia de aberraciones cromosómicas en los POEs versus la población general, sin embargo, se deberá esperar los resultados de la segunda fase de investigación, donde al ampliar la muestra en análisis se podrán obtener conclusiones estadísticamente significativas.

Palabras claves: Aberraciones cromosómicas, exposición ocupacional, radiación ionizante. 


\section{Citogenetic effects upon lymphocyes in subjects exposed to ionizing radiadion in an interventional laboratory cardiology: pilot study and literature review}

Background: There is growing evidence of an increased number of chromosomes aberrations in subjects exposed to low levels of ionizing radiation (POEs). There are few studies on this subject in Latin America

Aim: To evaluate the frequency of chromosome aberrations in lymphocytes obtained from peripheral blood in subjects working in laboratories where low levels of ionizing radiation are present and to compare these findings to those of unexposed subjects.

Methods: A cytogenic analysis to quantify chromosome aberrations was performed in 6 POs subjects from a cardiology invasive laboratory and 6 controls from a general unexposed population.

\section{Introducción:}

Los efectos biológicos de la radiaciones ionizantes según los tejidos irradiados se clasifican en efectos somáticos, que son aquellos que aparecen cuando los daños se manifiestan durante la vida del individuo irradiado y los efectos genéticos o hereditarios, que son aquellos en que los daños se manifestarían en la descendencia del individuo irradiado ${ }^{1}$.

Las radiones ionizantes (RI) tienen dos mecanismos a través de los cuales causan daño a nivel celular: los efectos directos, que proponen que la radiación actúa directamente sobre los átomos de las moléculas dentro de las células tales como enzimas, proteínas estructurales, y RNA, las cuales son vulnerables al daño por radiación. Sin embargo, el DNA es su principal objetivo, en el cual la radiación produce quiebres en los cromosomas singles o dobles ${ }^{2}$, lesiones que pueden ser reparadas o no. Es así como se originan posteriormente las denominadas
Results: Compared to controls, an approximately 6-fold increase in the number of chromosome aberrations was observed.in subjects exposed to ionizing radiation

Conclusion: These preliminary results indicate that there is an increased number of chromosome aberrations in subjects exposed to low levels of ionizing radiation, as occurs in people working in a cardiology interventional laboratory. Studies in large numbers of subjects and preferably followed prospectively are needed to evaluate more precisely this effect.

Keywords: Chromosome aberrations, ionizing radiation, occupational hazard. "aberraciones cromosómicas" (AC) y los "micro-núcleos". Las AC son el indicador biológico de actividad mutagénica más ampliamente desarrollado para evaluar la exposición a mutágenos y agentes carcinogenéticos ${ }^{3}$. Básicamente, el examen de $\mathrm{AC}$ consiste en observar al microscopio aberraciones de los cromosomas de linfocitos $\mathrm{T}$ de sangre periférica humana (dicéntricos, fragmentos acéntricos, rupturas, deleciones y otras anomalías). La importancia de cuantificar estas AC es que se ha documentado que podrían gatillar una inestabilidad genómica y posteriormente el desarrollo de cáncer. Por lo tanto, un biomarcador basado en el análisis de las aberraciones cromosómicas podría hacer posible la estimación del riesgo de contraer cáncer ${ }^{4}$.

De todos los trabajadores expuestos a fuentes de radiación artificial a nivel mundial, los que laboran en ambientes médicos representan el grupo más grande. Aunque los niveles de exposición de estos sujetos son bajos y, en 
cualquier caso, bajo los límites regulatorios de $20 \mathrm{mSv}$ al año. Sin embargo, los procedimientos diagnósticos realizados en medicina nuclear, cardiología/radiología intervencional podrían representar una fuente de exposición ocupacional relativamente alta ${ }^{5}$.

En la actualidad, los umbrales máximos de dosis para los POEs recomendados por los organismos internacionales están muy claros. De acuerdo con la Comisión Inter-nacional de Protección Radiológica (ICRP), los límites de dosis ocupacionales actuales se presentan en la Tabla 1. $\mathrm{Al}$ estar estas dosis bajo estos umbrales se crea una falsa sensación de seguridad sobre los eventuales efectos no deseados de la RI. Por ello es necesario llamar la atención sobre el hecho de que no se necesita sobrepasar un umbral para que se presenten los efectos estocásticos. La radiosensibilidad propia de cada individuo es muy variable y el uso inadecuado de los dosímetros físicos es un problema siempre latente, que hace que la dosimetría física por sí sola no dé una visión exacta del real impacto bilógico de las RI. Es aquí donde la dosimetría biológica (AC) complementa la información proporcionada por la dosimetría física entregando información sobre el efecto ya presente en el POE, independiente de la carga laboral y las medidas de radioprotección tomadas.

\begin{tabular}{|c|c|c|}
\hline Magnitud & Órgano & Límite de dosis anual \\
\hline Dosis efectiva (E) & Cuerpo entero & 20 mSv* \\
\hline \multirow[t]{3}{*}{ Dosis equivalente $(\mathrm{Ht})$} & Cristalino & $20 \mathrm{mSv}^{*}$ \\
\hline & Piel & $500 \mathrm{mSv}^{\mathrm{a}}$ \\
\hline & Manos y pies & $500 \mathrm{mSv}$ \\
\hline \multicolumn{3}{|c|}{$\begin{array}{l}\text { * Media aritmética en cinco años consecutivos, desde que no } \\
\text { exceda de } 50 \text { mSv en cualquier año. }\end{array}$} \\
\hline
\end{tabular}

\section{Materiales y Métodos:}

\section{Sujetos}

\section{Este es un estudio piloto.}

Se describió y comparó la frecuencia de aberraciones cromosómicas, observadas en linfocitos obtenidos de sangre periférica de 6 trabajadores que laboran en unidades de Hemodinamía del Hospital Dr. Gustavo Fricke. Ellas se compararon con la frecuencia de aberraciones cromosómicas observadas en 6 sujetos de la la misma localidad y características de los POEs, que no estuvieron expuestos a fuentes de radiación artificial. Los datos se obtuvieron de registros dosimétricos, una encuesta personal y de los análisis del laboratorio de Citogenética del Hospital Dr. Gustavo Fricke.

Este estudio se adhirió a los principios éticos de la declaración de Helsinki y fue aprobado por el Comité de Ética para la investigación humana de la Universidad Viña del Mar. Se obtuvo un consentimiento informado por escrito de todos los participantes antes de cualquier procedimiento.

\section{Análisis de Aberraciones Cromosómicas:}

En los POEs y en los sujetos controles se realizó una encuesta breve sobre su historial dosimétrico. Luego se extrajo $3 \mathrm{ml}$ de sangre periférica, en un tubo "Vacutainer" con heparina de Sodio como anticoagulante. Una vez extraída la muestra, se realizó un cultivo de linfocitos, empleando $250 \mu \mathrm{L}$ de muestra en un tubo Falcón estéril plástico de $15 \mathrm{ml}$ que contenía $10 \mathrm{ml}$ de medio de cultivo PBMAX $^{\mathrm{TM}}$ (Gibco), durante 72 horas a $37^{\circ} \mathrm{C}$. Pasado este período, se agregó $0,1 \mathrm{ml}$ Colcemid® (Gibco) por 1 hora y media, luego se centrifugó (Centrifuga JOUAN C312) por 10 minutos a $1200 \mathrm{rpm}$., eliminando el sobrenadante. Posteriormente, se suspendió el pellet con cloruro de potasio $(\mathrm{KCl}) 0,075 \mathrm{M}$ (Amresco) a $37^{\circ} \mathrm{C}$ mezclando con pipeta Pasteur y se llevó a baño termorregulado (Memmert modelo WNB 7-45) a $37^{\circ} \mathrm{C}$ por 9 minutos. Luego se agregó $2 \mathrm{ml}$ de fijador, se mezcló invirtiendo el tubo y se centrifugó por 10 minutos a $1200 \mathrm{rpm}$.

Se eliminó el sobrenadante, se agregó $8 \mathrm{ml}$ de fijador y se re-suspendió con pipeta Pasteur y se llevó a $-5^{\circ} \mathrm{C}$ por mínimo 1 hora. Posteriormente, se centrifugó por $10 \mathrm{mi}-$ nutos a 1200 rpm, se eliminó el sobrenadante y se agregó $5 \mathrm{ml}$ de fijador, re-suspendiendo con pipeta Pasteur. Se centrifugó por 10 minutos a 1200 rpm, se eliminó el sobrenadante y se re-suspendió el pellet con $1 \mathrm{ml}$ de fijador. Se dejó caer tres gotas sobre un portaobjetos esmerilado a una distancia de $40 \mathrm{~cm}$ y se colocó el preparado en estufa a $37^{\circ} \mathrm{C}$. Luego se tiñó con solución Giemsa a pH 6,8 durante 8 minutos, se enjuagó el portaobjeto con agua destilada y se dejó secar en estufa a $37^{\circ} \mathrm{C}$.

La visualización de las láminas se realizó en microscopio óptico (Olympus BX53) con objetivos de 10x y 100x. Para cada sujeto se analizaron mitosis $(\mathrm{n}=100)$ en placas metafásicas, buscando alteraciones tipo gap, fracturas, cromosoma en anillo, cromosoma dicéntrico, separación de cromátidas hermanas (SCE) y poliploidías.

Para conocer las dosis a las que estuvieron expuestos los POEs se registraron todas las dosimetrías disponi- 
bles, incluyendo registros en base a dosimetría fílmica y dosimetría con TLD.

\section{Análisis estadístico}

En este estudio piloto solo se presentan resultados descriptivos y una estimación de las diferencias en aberraciones cromosómicas. En una segunda fase, incorporando un número muy superior de sujetos y con mayor confiabilidad estadística se expresarán relaciones más detalladas y de mayor confiabilidad estadística.

\section{Resultados:}

Características demográficas y laborales sujetos controles y de POEs trabajando en Laboratorios de Hemodinamia (cardiología intervencional) y de Medicina Nuclear Las características basales de los sujetos investigados en este estudio piloto se describen en la Tabla 2. Llama la atención la gran cantidad de años que llevan expuestos a radiaciones ionizantes los POE (16,2 $\pm 0,7$ años), tiempo que además fue elaborado exclusivamente en un

\begin{tabular}{|c|c|c|}
\hline & $\begin{array}{c}\text { Profesionales } \\
\text { expuestos } \\
n=6\end{array}$ & $\begin{array}{c}\text { Público } \\
\text { general } \\
n=6\end{array}$ \\
\hline \multicolumn{3}{|l|}{ Genero } \\
\hline Masculino n, (\%) & $6,(100)$ & $1,(16,6)$ \\
\hline Femenino $\mathrm{n},(\%)$ & - & $5,(83,4)$ \\
\hline $\operatorname{Edad} \overline{\mathbf{X}} \pm \mathrm{DS}$ & $48,3 \pm 12,6$ & $40,2 \pm 11,3$ \\
\hline \multicolumn{3}{|l|}{ Profesión/oficio } \\
\hline Médico n, (\%) & $1,(16,6)$ & - \\
\hline Tecnólogo Médico n, (\%) & $3,(49,8)$ & $2^{* \star},(33,2)$ \\
\hline Enfermera $\mathrm{n},(\%)$ & - & - \\
\hline Paramédico n, (\%) & $2,(33,2)$ & - \\
\hline Guardia n, (\%) & - & $1,(16,6)$ \\
\hline Auxiliar de aseo $n,(\%)$ & - & $2,(33,2)$ \\
\hline Dueña de casa $n,(\%)$ & - & $1,(16,6)$ \\
\hline \multicolumn{3}{|l|}{$\begin{array}{l}\text { Años trabajando en Lab. } \\
\text { de Hemodinamia. }\end{array}$} \\
\hline Médico* & 38 & - \\
\hline Tecnólogo Médico $\overline{\mathrm{X}} \pm \mathrm{DS}$ & $14,2 \pm 4,2$ & - \\
\hline Paramédico $\overline{\mathrm{X}} \pm \mathrm{DS}$ & $8, \pm 6,4$ & - \\
\hline \multicolumn{3}{|c|}{$\begin{array}{l}\text { Estimación de dosis de raciación acumulada } \\
\text { (mSv) }\end{array}$} \\
\hline Médico* & 856,9 & - \\
\hline Tecnólogo Médico $\overline{\mathrm{X}} \pm \mathrm{DS}$ & $47,2 \pm 13,2$ & - \\
\hline Paramédico $\overline{\mathrm{X}} \pm \mathrm{DS}$ & $17,5 \pm 18,3$ & - \\
\hline RI: radiación ionizante & \multicolumn{2}{|c|}{ * Solo es un médico no aplica la DS } \\
\hline
\end{tabular}

solo tipo de unidad de radiología, la unidad de Hemodinamia del Hospital Dr. Gustavo Fricke.

En los Cariogramas de linfocitos de sangre periférica, se identificaron las siguientes aberraciones cromosómicas: GAP, fracturas de cromosomas, cromosomas acéntricos, (carecen de centrómero) y mitosis con poliploidía. Estas últimas se visualizaron en el Cariograma como un cambio o variación en el número normal de cromosomas (Figura 1).

Figura 1. Aberraciones cromosómicas observadas en los Cariogramas. En el paciente 1 se observa un gap. Las otras aberraciones corresponden al paciente 3 que presentó múltiples aberraciones, como fracturas, poliploidía y un fragmento acéntrico.

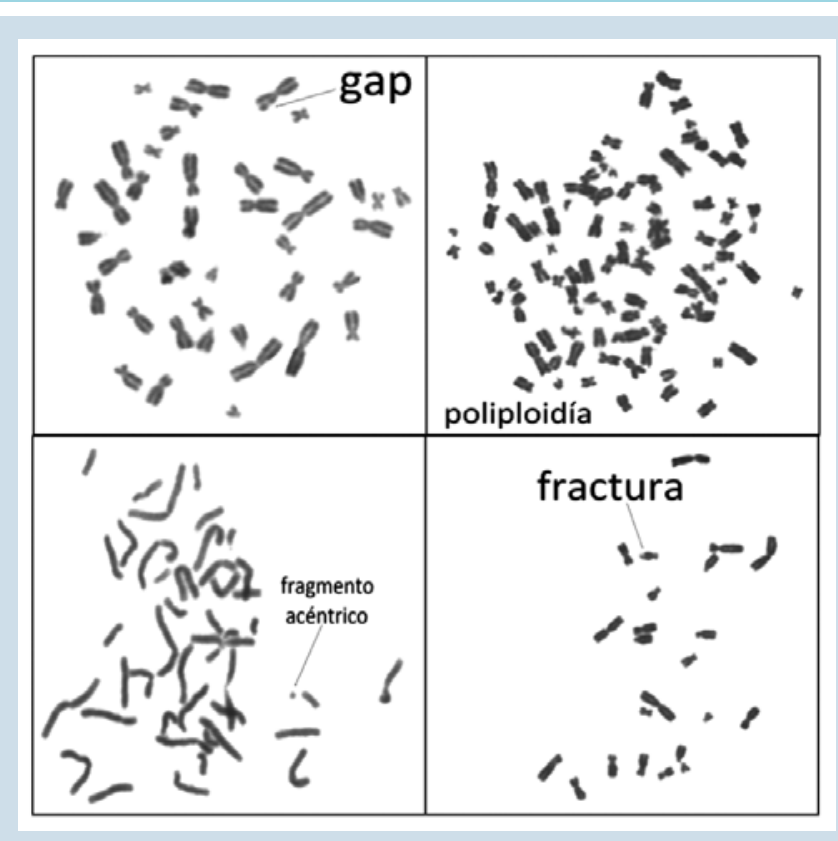

La frecuencia absoluta de células con aberraciones cromosómicas en linfocitos de sangre periférica de trabajadores expuestos a radiación y sujetos sanos de la población general se muestran en la Tabla 3. Un total de 100 metafases para cada sujeto fueron analizadas en busca de aberraciones cromosómicas, tales como gap, fracturas cromosómicas, cromosomas acéntricos, dicéntricos y mitosis poliploídia. La relación entre las frecuencias absolutas de AC en el grupo expuesto en relación al no expuesto fue de 6:1.

\section{Limitaciones}

Se constató que los registros de dosis de radiación fílmicos eran muy poco fiables por cuanto aparecen registros idénticos en los cuatro trimestres de varios años 


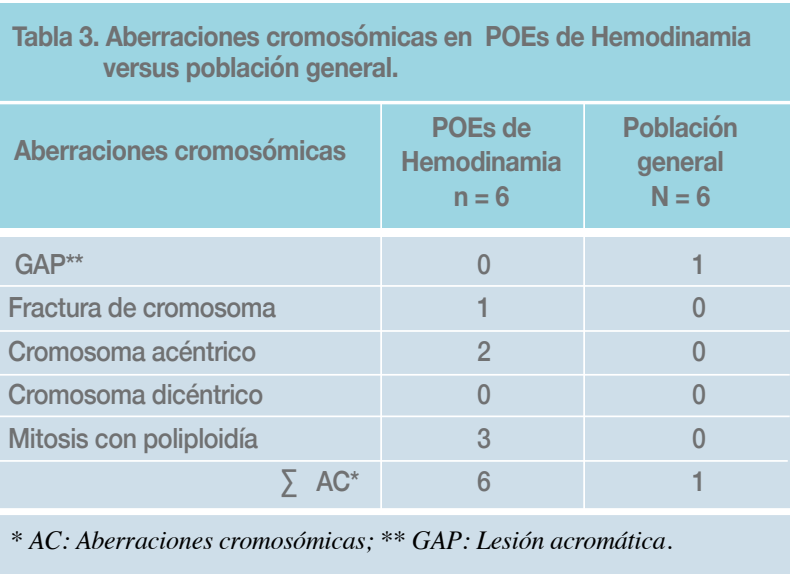

también aparecen muchos registros indicados como: PE (película extraviada), PND (película no devuelta) y NRD (no registra dosis). Por lo tanto, para conocer la dosis acumulada de vida se decidió extrapolar multiplicando el promedio anual de dosis registrada con los dosímetros TLD por el número total de años laborado.

\section{Discusión:}

Una revisión exhaustiva de los estudios reportados en la literatura científica los últimos 42 años, identificó 20 estudios de aberraciones cromosómicas en el contexto de unidades de radiología (Tabla 4), de los cuales $40 \%$ provienen de Europa, 30\% de Asia, 25\% de América y $5 \%$ de África. En cuanto a las unidades clínicas donde se llevaron a cabo estos estudios, se distribuyeron de la siguiente manera: 13 Unidades de Radiodiagnóstico 3,6-19, 4 Unidades de Medicina Nuclear ${ }^{11,12,14,16}, 4$ Unidades de Radioterapia ${ }^{4,11,14,16}, 4$ unidades de cardiología y angiografía intervencional ${ }^{8,10,20,21}, 1$ unidad de $\mathrm{Tac}^{4}$ y 1 Reactor Nuclear ${ }^{7}$.

De manera global, es decir, al considerar los 20 estudios analizados, estos concluyen en un 75\% 3,4,6-14,18,20,2224 que existiría una mayor frecuencia y/o incidencia de $\mathrm{AC}$ en los grupos expuestos en comparación con los controles. El presente estudio piloto sigue esta misma tendencia mostrando 6 veces más AC en los grupos expuestos en comparación con los controles. Dos de los 20 estudios revisados no fueron concluyentes en afirmar una mayor frecuencia y/o incidencia de AC en los grupos expuestos en comparación con los controles ${ }^{16}$ y 25 . En el primero se comparó 46 trabajadores expuestos, excluyéndose personas con enfermedades virales, metabólicas, aquellas que usaban medicamentos potencial-

Tabla $N^{\circ}$ 4. Datos de 20 estudios de Aberraciones cromosómicas en POEs expuestos a radiaciones ionizantes en ámbitos clínicos (publicados en la literatura desde 1987)

\begin{tabular}{|c|c|c|c|c|c|c|c|c|c|}
\hline Referencia & Pais & Aกั้ & $($ Exp./control) n & Unidad clinica & Tipo de Radiación & $\begin{array}{l}\text { Tipo de sujetos } \\
\text { estudiados }\end{array}$ & Tipo de estudio & Conclusion & $\begin{array}{l}\text { Nivel de evidencia de } \\
\text { Odford (OC冫BM) }\end{array}$ \\
\hline Bigatti et. at. & Italia & 1987 & $63 / 30$ & Radiodiagnostico & Rayos-x & $\begin{array}{l}\text { Medicos, Enfermeras } \\
\text { Técnicos paramedicos }\end{array}$ & Cohorte Retrospectiva & $>$ incidencia de AC en grupo expuesto a fl & $2 \mathrm{~b}$ \\
\hline Hagelstróm et. al. & Argentina & 1995 & $\begin{array}{l}10 / 10 \\
10 / 10\end{array}$ & $\begin{array}{l}\text { Radiodiagnostico } \\
\text { Med. Nuclear }\end{array}$ & $\begin{array}{l}\text { Rayos-X } \\
\text { Rayos-Y }\end{array}$ & $\begin{array}{l}\text { Medicos y Tecnicos } \\
\text { paramedicos }\end{array}$ & Cohorte Retrospectiva & $>$ incidencia de AC en grupo expuesto a fll & $2 \mathrm{~b}$ \\
\hline Pincheira et. al. & Chile & 1999 & $35 / 30$ & $\begin{array}{l}\text { Radiodiagnóstico } \\
\text { CCHEN }\end{array}$ & $\begin{array}{l}\text { Rayos-X } \\
\text { Rayos-Y } Y\end{array}$ & $\begin{array}{l}\text { Medicos, Tecnicos } \\
\text { paramedicos y }\end{array}$ & Cohorte Retrospectiva & $>$ incidencia de AC en grupo expuesto a fll & $2 \mathrm{~b}$ \\
\hline Cardoso et. al. & Brasil & 2001 & $8 / 8$ & $\begin{array}{l}\text { Med. Nuclear, Radioterapia y } \\
\text { Radiodiagnóstico }\end{array}$ & $\begin{array}{l}\text { Rayos-X } \\
\text { Rayos-Y }\end{array}$ & Trabajadores médicos & Transversal & > Frecuencia de AC en grupo expuesto a Rl & 4 \\
\hline $\begin{array}{l}\text { Vera Garaj-Vrhovac } \\
\text { et. al }\end{array}$ & Croacia & 2003 & $50 / 50$ & Radiodiagnóstico y Cirugia & Rayos-r & $\begin{array}{l}\text { Medicos, Tecnologos } \\
\text { Medicos y Enfermeras }\end{array}$ & Cohorte Retrospectiva & > Daño en el DNA del grupo expuesto a RI & $2 \mathrm{~b}$ \\
\hline Baquero et. al. & Colombia & 2004 & $15 / 0$ & $\begin{array}{l}\text { Med. Nuclear, Radioterapia, } \\
\text { Fisica Médica y }\end{array}$ & $\begin{array}{l}\text { Rayos-X } \\
\text { Rayos-Y } Y\end{array}$ & S/datos & Cohortes de baja calidad & $\begin{array}{l}\text { los datos "sugieren" una > frecuencia relativa } \\
\text { de AC }\end{array}$ & 4 \\
\hline Maffei et. al. & Italia & 2004 & $34 / 35$ & Medicos y Téenicos & Rayos- $x$ & Medicos y Téenicos & Cohorte Retrospectiva & > incidencia de AC en grupo expuesto a fl & $2 \mathrm{~b}$ \\
\hline Samavat and & Iran & 2004 & $65 / 65$ & Radiodiagnóstico & Rayos $-x$ & Tecnologas Médicos & Transversal & > Frecuencia de AC en grupo expuesto a RI & 4 \\
\hline Milacic Snezana & $\begin{array}{l}\text { Serbiay } \\
\text { Montenegro }\end{array}$ & 2004 & $46 / 22$ & S/datos & Rayos- $\mathrm{X}$ & Trabajadores médicos & Cohorte Retrospectiva & $\begin{array}{l}\text { RI podrian causar inestabilidad de los } \\
\text { cromosomas en personas radio-sensibles }\end{array}$ & $2 \mathrm{~b}$ \\
\hline Ali Shabestani & $\operatorname{Iran}$ & 2006 & $62 / 29$ & Radiodiagnostico & Rayos-X $x$ & $\begin{array}{l}\text { Trabajadores de } \\
\text { unidad de radiologla }\end{array}$ & Transversal & > Frecuencia de AC en grupo expuesto a RII & 4 \\
\hline Dias et al. & Brasal & 2007 & $36 / 36$ & Radiodiagnostico & Rayos-X & Médicos y Técnicos & Cohorte Retrospectiva & $\begin{array}{l}\text { Sin diferencias significativas de las AC en grupo } \\
\text { expuesto a fl versus el No-expuesto. }\end{array}$ & $2 \mathrm{~b}$ \\
\hline $\begin{array}{l}\text { Griciene and } \\
\text { Slapsyte }\end{array}$ & Lituania & 2007 & $70 / 70$ & $\begin{array}{l}\text { Radiodiagnosticoy } \\
\text { empleados de Reactor }\end{array}$ & Rayos $-x$ & $\begin{array}{l}\text { Trabajadores de } \\
\text { Reactor, Medicos } y\end{array}$ & Cohorte Retrospectiva & $\begin{array}{l}\text { > Frecuencia de AC en grupo expuesto a RI de } \\
\text { unidad de Radiología No paso lo mismo con el }\end{array}$ & $2 \mathrm{~b}$ \\
\hline Kasuba et. al. & Croacia & 2008 & $765 / 200$ & $\mathrm{~S} /$ datos & Rayos- $x$ & Trabajadores médicos & Cohorte Retrospectiva & $>$ Frecuencia de AC en grupo expuesto a Ri & $2 \mathrm{~b}$ \\
\hline Zakeriet. Al. & Iran & 2010 & $37 / 37$ & Cardiología intervencional & Rayos $-\bar{x}$ & Cardiologos & Cohorte Retrospectiva & > Frecuencia de AC en grupo expuesto a RI & $2 \mathrm{~b}$ \\
\hline Sakly et. A. & Tunez & 2012 & $60 / 27$ & $\begin{array}{l}\text { Cardiologia intervencional y } \\
\text { Radiodiagnóstico }\end{array}$ & Rayos- $x$ & S/datos & Cohorte Retrospectiva & > Frecuencia de AC en grupo expuesto a RI & $2 \mathrm{~b}$ \\
\hline Saberit. al. & Iran & 2013 & $33 / 11$ & $\begin{array}{l}\text { Radioterapia, TACy } \\
\text { Angiografia }\end{array}$ & Rayos-X & Tecnicos & Cohorte Retrospectiva & $\begin{array}{c}\text { > Frecuencia de AC en grupo expuesto a RI de } \\
\text { Angiografia }\end{array}$ & 4 \\
\hline Santovito et. al. & Italia & 2014 & $21 / 21$ & Radiodiagnóstico & Rayos $-\bar{x}$ & Tecnicos & Cohorte Retrospectiva & > Frecuencia de AC en grupo expuesto a RI & $2 \mathrm{~b}$ \\
\hline Vellingiri et. AL. & India & 2014 & $56 / 56$ & $\begin{array}{l}\text { Radiodiagnóstico, Cardiologia } \\
\text { y laboratorios de Ortopedia }\end{array}$ & Rayos-X & $\begin{array}{l}\text { Medicos, Técnicos } y \\
\text { Enfermeras }\end{array}$ & Cohorte Retrospectiva & $\begin{array}{l}\text { > Frecuencia de AC en grupo expuesto a RI, } \\
\text { especialmente en los Cardiologos. }\end{array}$ & $2 \mathrm{~b}$ \\
\hline Qian et. A. & China & 2016 & $1392 / 143$ & Entorno Sanitario & Rayos- $x$ & S/datos & Cohorte Retrospectiva & > Frecuencia de AC en grupo expuesto a RI & $2 \mathrm{~b}$ \\
\hline Kumar et. Al. & India & 2016 & $83 / 51$ & $\begin{array}{l}\text { Radiodiagnóstico, } \\
\text { Radioterapia y Medicina } \\
\text { Nuclear }\end{array}$ & $\begin{array}{l}\text { Rayos-X, Rayos-Y } \\
\text { Y Rayos- } \beta\end{array}$ & $\begin{array}{l}\text { Tecnologos Medicos y } \\
\text { Tecnicos }\end{array}$ & Cohorte Retrospectiva & > Frecuencia de AC en grupo expuesto a RI & $2 \mathrm{~b}$ \\
\hline
\end{tabular}

Exp: expuestos; OCEBM: Oxford Centre for Evidence Based Medicine; AC: aberraciones cromosómicas; RI: radiación ionizante; CCHEN: Comisión Chilena de Energía Nuclear. 


\begin{tabular}{|c|c|c|c|c|c|c|c|}
\hline Referencia & País & Año & n (Exp/Con) & Tipo estudio & Aleatorización & $\begin{array}{l}\text { Cegamiento } \\
\text { del análisis }\end{array}$ & $\begin{array}{l}\text { Nivel de } \\
\text { evidencia de } \\
\text { OCEBM }\end{array}$ \\
\hline Zakeri et. Al(20). & Irán & 2010 & $37 / 37$ & Cohorte Retrospectiva & No & No & $3 b$ \\
\hline Sakly et. $A^{l(8)}$. & Túnez & 2012 & $60 / 27$ & Cohorte Retrospectiva & No & No & $3 b$ \\
\hline Saberi t. al ${ }^{(21)}$. & Irán & 2013 & $33 / 11$ & Cohorte Retrospectiva & No & No & 4 \\
\hline Balanchandar et. Al(10). & India & 2014 & $56 / 56$ & Cohorte Retrospectiva & No & No & $3 b$ \\
\hline
\end{tabular}

mente inductores de AC y fumadores, y se compararon con 22 trabajadores no expuestos. Lamentablemente, los autores no dan cuenta ninguna comparación estadística entre grupos; adicionalmente, no se especifica exactamente en qué área de la Radiología se desempeñaban los POEs. información relevante porque los niveles de exposición están en directa relación con el área de desempeño, siendo las unidades de Cardiología intervencional las que se asocian con mayores niveles de exposición ${ }^{26}$. A su vez, el estudio de Baquero también excluyó a pacientes con potenciales condiciones inductoras de $\mathrm{AC}$, no fue un estudio comparativo, solo se siguió una cohorte de POEs a radiación ionizante sin detallar el área específica en que laboraban.

El único estudio que no encontró diferencias significa- tivas en la frecuencia de AC entre el grupo expuesto y el grupo control es un estudio brasileño que incluyo 35 personas expuestas, pero no especifica las unidades de trabajo lo que impide inferir la magnitud de la exposición a radiación.

En solo 4 estudios se trató de exposición a RI en laboratorios de cardiología intervencionista (Tabla 5), Todos ellos fueron del tipo de cohortes retrospectivas. En la Figura 2 se puede apreciar que todos los estudios muestran gráficamente una mayor frecuencia de $\mathrm{AC}$ de los grupos expuestos en relación a los controles.

Según sus autores todas estas diferencias son estadísticamente significativas, basadas fundamentalmente en test no paramétricos. El nivel de evidencia de estos estudios correspondió solo a 3b a 4 (Centro de Medicina

Figura 2. Porcentaje de Aberraciones Cromosómicas en POEs de unidades de Cardiología Intervencional en distintas publicaciones.

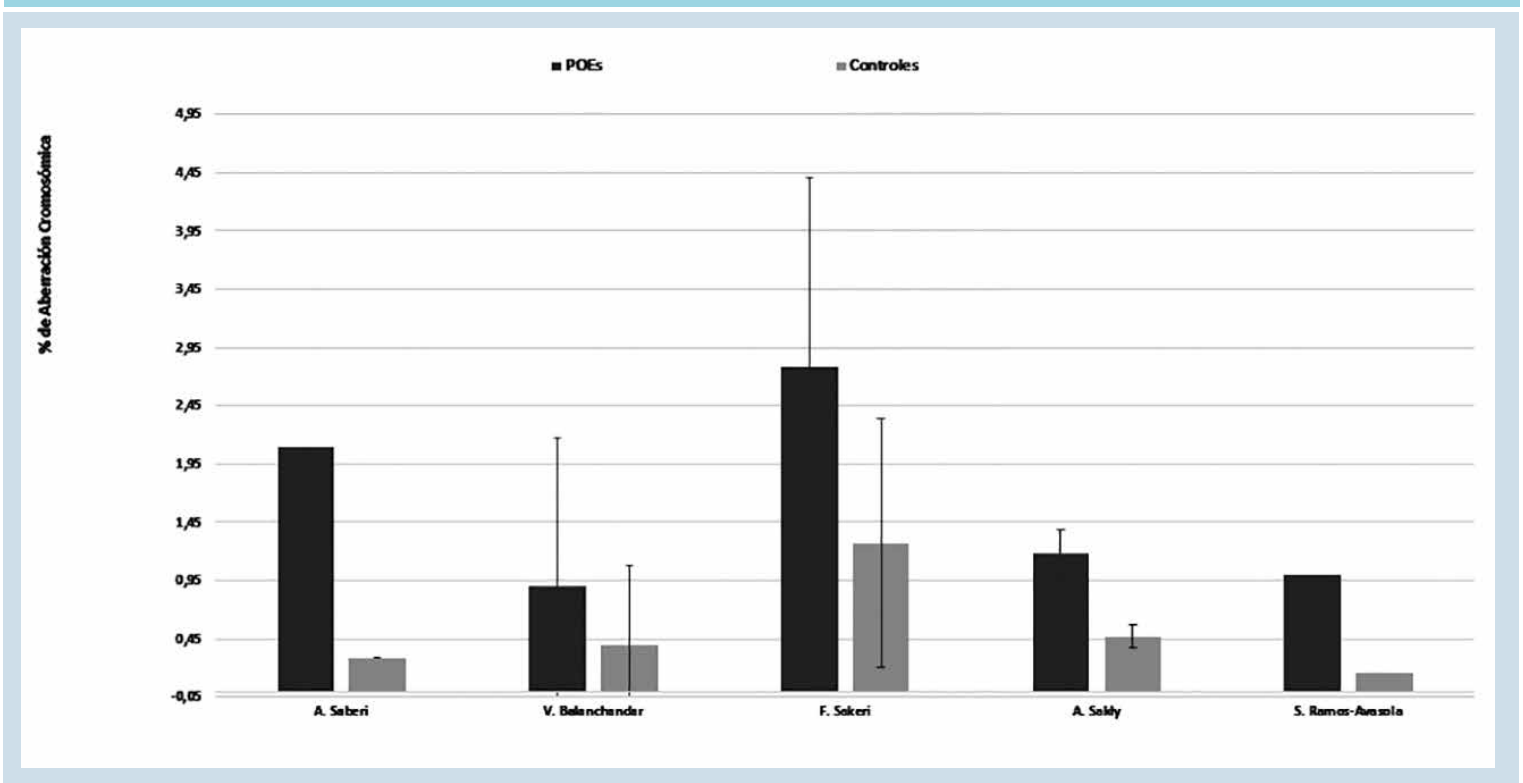


Basada en Evidencia, Oxford).

En general, el conjunto de evidencia de los estudios mencionados, concordando con el aquí presentado inducen a recomendar una acción preventiva dado que los efectos negativos de la RI son al menos moderados. Se hace imprescindible realizar nuevos estudios que agreguen un mayor nivel de evidencia con un adecuado manejo de todas las variables confundentes, tanto conocidas, como desconocidas que pudieran influir en los resultados. Ello implica necesariamente la realización de estudios prospectivos de tamaño muestral adecuado y a partir de muestras aleatorias. No obstante, es un hecho conocido que en estas unidades trabaja poco personal, haciendo necesario un estudio multicéntrico. De ser efectiva y confirmada la relación causal, obligaría a tomar medidas inmediatas de carácter preventivo y/o de vigilancia epidemiológica y así disminuir el riesgo latente de cáncer. Una de las medidas más eficaces sería la implementación de un sistema de vigilancia epidemiológica en base a dosimetría biológica cuantificando las AC y estableciendo el recuento de micronúcleos. esta propuesta ya ha sido sugerida en otro estudio realizado en Bélgica 5 .

\section{Conclusiones:}

Este estudio apoya el efecto negativo de la radiación ionizante sobre los cromosomas de linfocitos circulantes. Preliminarmente, sugiere que tales anormalidades son, aproximadamente, 6 veces más frecuentes en sujetos expuestos a RI que en controles. Más información deberá obtenerse con un estudio multicéntrico prospectivo y con un número significativamente mayor de sujetos.

\section{Referencias}

1. VIVALLO L, VILLANUEVA L, SANHUEZA S. Efectos de las Radiaciones Ionizantes en el ser Humano. Comisión Chilena de Energía Nuclear, Santiago; 2010.

2. BEFORE R, COME Y, Introduction A, Protection R. 13. Biological Effects of Ionizing Radiation. In 2010. p. 1-12.

3. SAMAVAT H, MOZDARANI H. Chromosomal aberrations in Iranian radiation workers due to chronic exposure of X-irradiation. Int J Low Radiat. 2004;1(2):216.
4. SABERI A, SALARI E, LATIFI SM. Cytogenetic analysis in lymphocytes from radiation workers exposed to low level of ionizing radiation in radiotherapy, CT-scan and angiocardiography units. Mutat Res Toxicol Environ Mutagen. 2013 Jan;750(1-2):92-5.

5. VRAL A, DECORTE V, DEPUYDT J, WAMBERSIE A, THIERENS H. A semi-automated FISH-based micronucleus-centromere assay for biomonitoring of hospital workers 
exposed to low doses of ionizing radiation. Mol Med Rep. 2016;14(1):103-10.

6. BIGATTI P, LAMBERTI L, ARDITO G, ARMELLINO F. Cytogenetic monitoring of hospital workers exposed to low-level ionizing radiation. Mutat Res Toxicol. 1988;204(2):343-7.

7. GRICIENĖ B. Assessment of chromosomal aberrations in workers chronically exposed to ionising radiation. 2007;53(4):5-10.

8. SAKLY A. Assessment of Chromosomal Aberrations and Micronuclei in Peripheral Lymphocytes from Tunisian Hospital Workers Exposed to Ionizing Radiation. 2012;00(00):1-6.

9. SANTOVITO A, CERVELLA P, DELPERO M. Increased frequency of chromosomal aberrations and sister chromatid exchanges in peripheral lymphocytes of radiology technicians chronically exposed to low levels of ionizing radiations. Environ Toxicol Pharmacol. 2014;37(1):396-403.

10. VELLINGIRI B, SHANMUGAM S, DEVI M, ANAND S, N DS, CHO S, et al. Cytogenetic endpoints and Xenobiotic gene polymorphism in lymphocytes of hospital workers chronically exposed to ionizing radiation in Cardiology , Radiology and Orthopedic Laboratories. 2014;100:266-74.

11. KUMAR D, KUMARI S, SALIAN SR, UPPANGALA S, KALTHUR G, CHALLAPALLI $S$, et al. Genetic Instability in Lymphocytes is Associated with Blood Plasma Antioxidant Levels in Health Care Workers Occupationally Exposed to Ionizing Radiation. Int J Toxicol. 2016;35(3):327-35.

12. HAGELSTRÖM AH, GORIA NB, LARRIPA IB. Chromosomal damage in workers occupationally exposed to chronic low level ionizing radiation. Toxicol Lett. 1995;76(2):113-7.

13. PINCHEIRA J, LÓPEZ I, SANHUEZA S, FERRUZ P, NAVARRETE M, SANTOS M, et al. G2 repair and chromosomal damage in lymphocytes from workers occupationally exposed to low-level ionizing radiation. Biol Res. 1999;32(4):297-306.

14. CARDOSO RS, TAKAHASHI-HYODO S, PEITL P, GHILARDI-NETO T, SAKAMOTO-HOJO ET. Evaluation of chromosomal aberrations, micronuclei, and sister chromatid exchanges in hospital workers chronically exposed to ionizing radiation. Teratog Carcinog Mutagen. 2001;21(6):431-9.

15. GARAJ-VRHOVAC V, KOPJAR N. The alkaline Comet assay as biomarker in assessment of DNA damage in medical personnel occupationally exposed to ionizing radiation.Dans les dernières années, l'électrophorèse sur gel de cellule unique (SCGE) ou test des comètes a été largement utilisé po. Mutagenesis. 2003;18(3):265-71.
16. PULIDO HB, PARDO GG, SUÁREZ MG, MARINA L, SOTO O. Aberraciones cromosómicas en trabajadores expuestos a radiaciones ionizantes. Rev Cienc Salud Bogota. 2004;2(1):8-14.

17. CAI Y, YANG J, PANG H, KITAHARA M. Lactococcus fujiensis sp. nov., a lactic acid bacterium isolated from vegetable matter. Int J Syst Evol Microbiol. 2011;61(7):1590-4.

18. MONFARED AS, MOZDARANI H, SAMAVAT H, HASHEMOGHLI A. Chromosomal aberrations in radiation workers of radiology departments in Northern Iran-Babol. Int J Low Radiat. 2015;3(1):83.

19. DIAS FL, ANTUNES LMG, REZENDE PA, CARVALHO FES, SILVA CMD, MATHEUS JM, et al. Cytogenetic analysis in lymphocytes from workers occupationally exposed to low levels of ionizing radiation. Environ Toxicol Pharmacol. 2007;23(2):228-33.

20. ZAKERI F, HIROBE T, NOGHABI KA. Biological effects of low-dose ionizing radiation exposure on interventional cardiologists. Occup Med (Chic Ill). 2010;60(6):464-9.

21. SABERI A, SALARI E, MAHMOOD S. Mutation Research / Genetic Toxicology and Environmental Mutagenesis Cytogenetic analysis in lymphocytes from radiation workers exposed to low level of ionizing radiation in radiotherapy , CTscan and angiocardiography units. Mutat Res - Genet Toxicol Environ Mutagen [Internet]. 2013;750(1-2):92-5. Available from: http://dx.doi.org/10.1016/j.mrgentox.2012.10.001

22. MAFFEI F, ANGELINI S, FORTI GC, VIOLANTE FS, LODI V, MATTIOLI S, et al. Spectrum of chromosomal aberrations in peripheral lymphocytes of hospital workers occupationally exposed to low doses of ionizing radiation. Mutat Res - Fundam Mol Mech Mutagen. 2004;547(1-2):91-9.

23. KAŠUBA V, ROZGAJ R, JAZBEC A. Chromosome aberrations in peripheral blood lymphocytes of Croatian hospital staff occupationally exposed to low levels of ionising radiation. Arh Hig Rada Toksikol. 2008;59(4):251-9.

24. QIAN QZ, CAO XK, SHEN FH, WANG Q. Effects of ionising radiation on micronucleus formation and chromosomal aberrations in Chinese radiation workers. Radiat Prot Dosimetry. 2016;168(2):197-203.

25. MILACIC S. Frequency of chromosomal lesions and damaged lymphocytes of workers occupationally exposed to $\mathrm{X}$ rays. Health Phys. 2005;88(4):334-9.

26. UNSCEAR. United Nations Scientific Committee on Effects of Atomic Radiation (UNSCEAR 2000). Vol. 79, Health Phys. 2000. 314 p. 